\title{
POST REGISTRATION COURSE
}

\section{CHEST COURSE}

A Post Registration Course on "Chests" organised by the Northern Transvaal Branch of the South African Society of Physiotherapy was held in the Physiotherapy School. Pretoria Hospital, on Friday and Saturday, May 27th and 28th. Sixty-one members and twenty-six student members of the Sol.th African Society of Physiotherapy attended the course, many coming from as far as Cape Town, Kimberley, East London, Durban, Pietermaritzburg, Bloemfontein, Barberton and many more towns in the Transvaal.

The course was opened by Dr. P. N. Swanepoel, Superintendent of the Pretoria General Hospital, who welcomed the visitors, spoke of the important part played by the Physiotherapist in all Chest work and particularly the necessity of them being a member of any Surgical Chest Unit.

On the first day of the course, lectures were given by:Dr. L. M. Jonck, Senior Lecturer in Anatomy, Pretoria University.

Dr. C. J. Labuschagne, Senior Lecturer in Physiology, Pretoria University.

Dr. L D. Erasmus, member of the Faculty of Medicine, Pretoria University.

Dr. J. C. v. d. Spuy, Senior Chest Surgeon, Pretoria Hospital, who also showed a very wonderful film on "Open Heart Surgery".

The second day of the course was given up entirely to the treatment of Chest Conditions by Physiotherapy. Miss M. White, M.C.S.P., Lecturer in the Department of Physiotherapy, Cape Town University, gave all the lectures and demonstrations.

Miss White's lectures and demonstrations were of the highest quality-clear and definite, she covered a tremendous amount of ground, devoting the morning session to medical and the afternoon to surgical conditions, and using Physiotherapy students and real patients to illustrate her points.

The Northern Transvaal Branch would like to express their thanks to Miss M. McDowell, M.C.S.P., Staff of the Physiotherapy Department of the Brompton Chest Hospital, London, England, for very kindly lending her own films which Miss White used to demonstrate the treatments of the asthmatic, individually and in groups.

In addition to the lectures and demonstrations two large rooms were set aside. One a specimen room, gave an excellent display of X-rays of Chest conditions, photographs of cases demonstrations incision lines position of drainage tubes, typical postures, etc., of post-operative chest conditions. Also many Anatomical and Pathological specimens of lungs were displayed.

In the second room were on view modern and up-to-date apparatus and also a book shop with the latest publications and pamphlets on Chest Conditions and many other medical books. In their free periods the members had an opportunity of meeting in these rooms and discussing not only Chest conditions but also other Physiotherapy matters.

We should like to express our thanks to all who co-operated so fully and helped to make the course such a success: to Dr. Swanepoel for his support, to all the Lecturers, to the patients and students who acted as models for Miss White, to the Staff of the X-ray Department for the loan of films and viewing boxes, to Mr. Marais and the Staff of the Photographic Department for the interest and trouble they took to give us such excellent photographs, to the doctors for the loan of specimens, to the School Staff, particularly the Secretary, Mrs. Smith, for all the "behind the scenes work" which helped in the smooth running of the course, and lastly, to the students who worked so hard and served all the meals with efficiency and charm.

\section{ASPECTS OF RESPIRATION}

\author{
By Dr. C. J. LABUSCHAGNE, M.Sc., Ph.D. \\ Senior Lecturer, Department Physiology, University of Pretoria. \\ A Lecture given to Delegates and Members of the South African Society of Physiotherapy on the first day of the \\ "Chest Course", which was held at the Pretoria Hospital School of Physiotherapy, on May 27th and 28 th, 1960.
}

What causes an Inspiration?

The immediate cause of an inspiration is the fact that the thoracic cavity increases in size in all dimensions. Because the lungs are attached to the thoracic walls and upper surface of the diaphragm (visceral and parietal pleurae) they are stretched out to the extent that the thoracic cage enlarges. (Hence the importance of normal elasticity of the lungs.)

Since the pulmonary volume increases, the intra pulmonary pressure decreases (Boyle's Law_-pressure -inversely proportional to volume), and, the lungs being connected with the exterior via the air passages, air flows passively into them until intra pulmonary pressure $=$ atmospheric pressure.

The extent or volume of the inspiration is therefore dependant on;

(i) the extent of increase in the size of the thoracic cavity, and

(ii) elasticity of the lungs.

With normal, quiet (eupneic) breathing this volume amounts to $500 \mathrm{ml}$.- so-called tidal air. Abnormal conditions of the thorax, pleurae or reduced elasticity of the lungs will therefore affect the tidal volume.
What causes enlargement of the thoracic cage?

This is the result of the specific structure of its bony framework and the contraction of certain muscles the inspiratory muscles. Of these the diaphragm and external intercostals are the main ones concerned in eupheic breathing. Their contraction enlarges the thoracic cage in all dimensions.

The contribution of each of these two sets varies in different individuals and in the two sexes. Generally the diaphragm accounts for $60 \%$ of the inspired air-diaphragmatic breathing - and the intercostals for the remaining $40 \%$ - costal breathing.

With a forced inspiration additional muscles come into play:

(i) NECK —scaleni

(ii) SHOULDER - pectoralis

A deep inspiration is the result of contraction of all the inspiratory muscles, rather than of a specific one or group. When certain inspiratory muscles are paralyzed (e.g. diaphragm) others come into play and respiration is maintained in some patients chiefly by the neck and shoulder muscles 
and in others almost solely by the abdominal muscles. It is indeed surprising how few muscles can maintain adequate ventilation of the lungs in eupneic breathing.

The inspiratory volume will therefore further depend on:

(i) the degree of contraction of a specific muscle, and

(ii) the number of inspiratory muscles contracting.

Maximal contraction of all inspiratory muscles causes maximal increase in the size of the thoracic cavity with resultant maximal inspiratory volume. This normally consists of:

(i) $500 \mathrm{ml}$. tidal air,

(ii) $2,500 \mathrm{ml}$. inspiratory reserve air.

Why do the inspiratory muscles contract?

This happens because nerve impulses conducted to them along the respiratory nerves, are transferred to the muscle fibres. A nerve impulse is a physico-chemical disturbance propagated at high velocity along the nerve fibre and is of the nature of an electric current.

The degree of contraction therefore depends on:

(i) the number of muscle fibres (in a muscle) receiving impulses and hence contracting, and

(ii) the number of muscles contracting.

The above two factors are conditioned by:

(i) the total number of impulses reaching a muscle per unit of time, and

(ii) the frequency of the impulses.

Nerve impulses are discharged by nerve cells or neurons. With eupneic breathing the discharge of impulses by respiratory neurons is asynchronous, i.e., some neurons discharge actively whilst others are quiescent. Obviously then, the greater the number of discharging neurons, the greater the number of contracting muscle fibres and muscles.

Nerve impulses are always discharged in trains or volleys. Within a volley impulses follow each other at a specific frequency, which may vary between $5-100 / \mathrm{sec}$. The higher the frequency, the more powerful the ensuing contraction. With eupneic breathing only portion of the available neurons discharge at relatively low frequency. With a maximal inspiratory effort, all available neurons discharge at maximum frequency.

Where do the impulses come from?

The immediate source of the impulses are the motor neurons in the anterior horns of the spinal cord segments $\mathrm{C} 3$, 4 and 5 and $\mathrm{T} 2-6$. 1mpulses reaching these neurons are relayed by them to the diaphragm along the phrenic nerve (C3,4 and 5) and to the intercostal muscles along the intercostal nerves (T2-6).

However, these motor neurons simply relay impulsesthey do not generate impulses. Generation of respiratory impulses is a function of neurons situated higher up in the brain stem, viz. the medulla oblongata and pons. These neurons possess the ability of continuous automatic, repetitive impulse generation, the resulting impulses being conducted from the brain stem to the motor neurons concerned in the spinal cord along the reticulo spinal tract.

The number of respiratory neurons discharging impulses and the frequency of their discharge therefore ultimately determine the degree of inspiration. These factors are in turn, determined by the sum total of all influences impinging upon the respiratory neurons. Such influences may be of two fold nature:

(i) chemical;

(ii) nervous.

Chemical.

The slightest chemical change in the environment of the respiratory neurons (blood interstitial fluid, intracellular fluid) alters their pattern of impulse generation and discharge.

Increased $\mathrm{pCO}_{2}$ or acidity activates more respiratory neurons and increases the frequency of their discharge with resulting deeper inspiration. Conversely decreaded $-\mathrm{pCO}_{2}$ or increased alkalinity leads to a shallow inspiration or apnoea-(temporary cessation of breathing). In this latter instance the respiratory neurons discharge no impulsesthey are inhibited.
Nervous.

The respiratory neurons are constantly bombarded by nerve impulses originating elsewhere within the central nervous system or peripheral organs.

A deep inspiration can be made voluntarily or the breath can be held, i.e. no inspiration made at all. Such excitatory or inhibitory impulses bombard the respiratory neurons from the cerebral cortex and are conducted to the brain stem along the extra--pyramidal system.

The sobbing inspiration of grief is the result of impulses originating in the cerebral cortex and hypotholamus.

Similarly, the changed inspiratory pattern in other activities, e.g. talking, singing, sneezing, coughing, sighing, etc., is the result of nervous bombardment of the respiratory neurons from the nerve centres concerned.

Stepping under a cold shower causes a sudden deep inspiration-gasp-due to peripheral impulses from the skin reaching the respiratory neurons and greatly increasing their activity.

What is the respiratory centre?

This is the name given to all those neurons concerned with the initiation and maintenance of respiration and located within the lower part of the brain stem, i.e. pons and medulla.

They are spread diffusely throughout the medulla and lower part of the pons and it is doubtful whether they form an anatomic unit. However, their main function is not only to produce an inspiration, but to maintain rhythmic breathing i.e., inspiration-expiration; inspiration-expiration, etc.

The respiratory centre is therefore subdivided into:

(i) inspiratory (apneustic) centre,

(ii) expiratory centre,

(iii) pneumotaxic centre.

Again these are more of physiological than anatomical units.

The inspiratory centre (inspiratory neurons) is responsible for maintained inspiration (apneusis)-as described. The expiratory and pneumotaxic centres, together with the so-called vagal mechanism, are responsible for expirationto be described now.

What causes an expiration?

The thoracic cavity decreases in size; the elastic lungs recoil and decrease in volume; the intrapulmonary pressure is raised to above atmospheric pressure and air flows out along the respiratory passages.

This decrease in the size of the thoracic cage is due to relaxation of the inspiratory muscles (in eupneic breathing). The expiratory volume is therefore $=$ inspiratory volume $=$ tidal air $=500 \mathrm{ml}$.

However, with a forced expiration, active contraction of the expiratory muscles occurs:

(i) internal intercostals,

(ii) abdominal muscles.

These muscles receive their impulses from the expiratory centre along the same route as that described for the inspiratory impulses.

With maximal contraction of the expiratory muscles, i.e. maximal decrease in the size of the chest cavity, the expiratory volume amounts to $1,500 \mathrm{ml}$. This consists of:

(i) tidal air $=500 \mathrm{ml}$.

(ii) expiratory reserve air $=1,000 \mathrm{ml}$.

The vital capacity therefore consists of:

(i) inspiratory reserve air $=2,500 \mathrm{ml}$.

(ii) tidal air $=500 \mathrm{ml}$.

(iii) expiratory reserve air $=1,000 \mathrm{ml}$.

$$
4,000 \mathrm{ml} \text {. }
$$

Factors determining the volume of the vital capacity therefore are:

(i) Size of the thoracic cage (cf. men and women);

(ii) Expandibility of the thoracic cage, i.e. normal structure -and function of its bony framework; 


\section{ASPECTS OF RESPIRATION (Continued from Page4).}

(iii) Strength (size) of the respiratory muscles-inspiratory and expiratory (cf. the respiratory muscles of the trained athlete);

(iv) Elasticity of the lungs (cf. the reduced vital capacity in all forms of fibrosis of the lungs);

(v) Functional efficiency of the nervous structures concerned:

(a) respiratory centre,

(b) anterior motor neurons (cf. poliomyelitis),

(c) peripheral efferent or motor nerves to the respiratory muscles.

\section{Why do the inspiratory muscles relax?}

Because they receive no impulses from the spinal cord motor neurons, which in turn receive no impulses from the inspiratory centre. Thus, the continuous discharge of repetitive impulses by the inspiratory neurons has temporarily been stopped or inhibited, or inspiration has been cut short to allow expiration to take place.

The responsible mechanisms for this are:

(i) pneumotaxic centre,

(ii) expiratory centre,

(iii) Hering-Breuer reflex (vagal mechanism).

\section{Pneumotaxic Centre.}

This consists of respiratory neurons scattered in the formatio reticularis of the pons. They make synaptical contact with those of the inspiratory and expiratory centres.

As the inspiratory neurons discharge to the anterior motor neurons, there is a simultaneous discharge to the pneumotaxic neurons. These in turn discharge to the expiratory neurons. At a certain critical level of excitation, the latter emit a barrage of impulses at the inspiratory neurons, which for a short period, completely inhibits their discharge.

\section{Hering-Breuer reflex.}

The delicate alveolar membranes of the lung contain receptors sensitive to the amount of stretch of inflation of the lung-stretch receptors.

As a lung becomes progressively more inflated during inspiration, these receptors are stimulated and a stream of afferent impulses is conducted along afferent vagus fibres to the expiratory neurons, which again inhibit the inspiratory neurons, allowing expiration to take place.

The rhythm of breathing is therefore the result of the inter action of the inspiratory, expiratory and pneumotoxic centres and the vagal mechanism.

Whereas the depth of an inspiration is determined by the degree of discharge by the inspiratory neurons the rate of respiration is dependent on the activity of the pneumotaxic and vagal mechanisms.

\section{How is respiration regulated?}

The respiratory pattern (i.e. depth and rate) is regulated, via the respiratory centre, by:

(i) chemical substances, and

(ii) nerve impulses.

\section{Chemical Substances.}

Under all conditions of normal, eupneic breathing, the respiratory pattern is regulated almost exclusively by the $\mathrm{CO}_{2}$ content of the Arterial blood. This is mediated through a direct action of the $\mathrm{HCO}_{3}$ (bicarbonate) ions on the respiratory neurons and changes in acidity $(\mathrm{pH})$ caused by increased or decreased $\mathrm{CO}_{2}$.
The normal tension of $\mathrm{CO}_{2}\left(\mathrm{pCO}_{2}\right)$ in arterial blood $=$ $40 \mathrm{~mm}$. Hg. Increasing this volume by as little as $2 \mathrm{~mm}$. $\mathrm{Hg}$. is sufficient to double pulmonary ventilation (hyperpnoea); hence the hyperpnoea of acidosis. Similarly a slighi decrease of this value causes apnoea; hence the slow shallow breathing of alkalosis.

In certain cases of anoxia, the respiratory pattern is controlled by the $\mathrm{O}_{2}$ content $\left(\mathrm{pO}_{2}\right)$ of the arterial blood, via the chemoreceptors of the aortic and carotid bodies.

\section{Nerve Impulses.}

These have already been referred to elsewhere.

The hyperpnoea of muscular activity is largely the effect of afferent impulses from the contracting muscles and moving joints of the respiratory centre.

During other physiological activities, e.g. talking, sneezing, coughing, swallowing, etc., the basic respiratory pattern (as controlled by the $\mathrm{CO}_{2}$-content of the arterial blood) is modified by nerve impulses from the respective nerve centres, acting on the neurons of the respiratory centre.

\section{PULMONARY TEST (Continlted from Page 7).}

reduction of timed vital capacity and the maximum breathing capacity. Residual volume is usually considerably increased both as an absolute value and as a proportion of the total lung capacity.

\section{Clinical Features.}

An advanced cause of emphysema will then be seen to have anoxaemia with cyanosis heightened frequently by secondary polycythaemia and shows the considerable ventilatory difficulty associated with hyperinflation of the lungs and diffuse expiratory obstruction. Clinically the patient wheezes on the slightest exertion or even at rest and has to use his accessory muscles of respiration. The chest becomes barrel-shaped and the ribs horizontally placed. Respiration is carried out partially by diaphragmatic excursion and "en bloc" movement of the barrel-shaped thoracic cage. On ausculation the prolonged expiratory difficulty of a forced maximal expiration is usually quite easily detected and in my opinion this is the most valuable single sign in the clinical assessment of the patient. For the most part also there is reduction in air entry as judged by the stethoscope.

\section{Treatment.}

Treatment is difficult and prolonged in most instances. It has to be said at the outset that emphysema as such cannot be influenced by treatment and any therapeutic attack is directed against bronchospasm, infection and viscid secretions with a variety of agents including antibiotics, antispasmodics, steroids, detergent and wetting inhalant and enzymes (streptokinase and trypsin).

Although there is not the slightest doubt in my mind that Physiotherapy is of considerable value in making more efficient use of the patient's natural capacity for ventilation, there is no objective improvement in pulmonary function tests in controlled series of patients in whom this has been measured before and after physiotherapy.

Complications (especially bronchopneumonia and corpulmonale) are of a serious nature and require vigorous treatment in their own right. Frequently then we have a patient who is very ill with grossly reduced respiratory reserve and considerable secondary infection in the lungs with associated cardiac failure and a wide, vigorous and persistent therapeutic attack is indicated. As far as future developments are concerned, however, prevention is likely to prove more rewarding than an attempt at cure. 